\title{
Artrodese do carpo em gatos associada à hidroxiapatita
}

\author{
Arthrodesis of the carpus in cats associeted with hydroxyapatite
}

\author{
Francisco de Assis Dórea Neto ${ }^{1}$ João Guilherme Padilha Filho ${ }^{2}$ Arianne Pontes Oriá ${ }^{1}$ \\ Juliana Maziero Furlani ${ }^{1}$ Luís Alberto dos Santos ${ }^{3}$
}

\section{- RELATO DE CASOS -}

\section{RESUMO}

Realizaram-se artrodeses carpais em dois gatos como forma de tratamento para alterações severas na articulação carpiana. A hidroxiapatita foi empregada para preenchimento do espaço articular do carpo. Os animais foram encaminhados ao Serviço de Ortopedia do Hospital Veterinário, UNESP, Campus de Jaboticabal, São Paulo - Brasil. O animal 1, Siamês, 10 anos de idade, apresentava apoio inadequado do membro torácico esquerdo, dor, hiperextensão, instabilidade, luxação, degeneração articular e osteartrite da articulação radiocárpica. $O$ animal 2, sem raça definida, 8 meses de idade, apresentava apoio inadequado do membro torácico direito, hiperflexão da articulação cárpica, contração dos tendões da região carpiana, rotação externa do olécrano e o membro voltado para dentro. Foram aplicados fixadores esqueléticos externos (tipo IIb) com barras de polimetilmetacrilato, em ambos os casos. As avaliações clínicas e radiográficas após os procedimentos, demonstraram que a hidroxiapatita é uma alternativa promissora como substituto para os enxertos ósseos.

Palavras-chave: artrodese, carpo, gato, hidroxiapatita, fusão óssea.

\section{ABSTRACT}

Severe disorders of the carpus in two cats were treated with arthrodesis. Hydroxyapatite was usage in filling up of the joint space in the carpus. Both cats were sent to the Orthopedic Service of the Veterinary Hospital, UNESP, Jaboticabal, São Paulo - Brazil. The first cat, a Siamese, 10 years old, showed hyperextension movements and luxation of the carpus joint and pain with inadequate support of the left thoracic limb. It was also observed joint degeneration and osteoarthritis. The second animal, a domestic shorthair, 8 months of age, showed inadequate support of the right thoracic limb with hyperflexion of the carpus joint and contractures of the carpus flexor tendons. A modified trans-articular external skeletal fixator (tipe IIb) with methylmethacrylate bars was utilized in these cases. The clinical and radiographic evaluations of the joints after the surgical procedure proved that the hydroxyapatite can be used as an promising alternative to cancellous bone grafts.

Key words: arthrodesis, cat, carpus, hydroxyapatite, bone fusion.

\section{INTRODUÇÃO}

Define-se artrodese como a remoção do movimento articular por procedimento cirúrgico e formação de unidade óssea sólida (LESSER, 1993; TURNER \& LIPOWITZ, 1996). É considerado procedimento relevante no salvamento do membro, sendo indicado para afecções dolorosas, instabilidade e perda da função articular (JOHNSON, 1995; TURNER \& LIPOWITZ, 1996).

As cerâmicas de fosfato de cálcio, em especial a hidroxiapatita, foram concebidas para a remodelação e reconstrução de defeitos ósseos. As propriedades de biocompatibilidade, bioatividade e osteocondução destas, permitem que possam ser implantadas em sítios ósseos, sem indução de resposta imunológica (LEGEROS, 1991). O objetivo deste relato foi demonstrar a utilização da hidroxiapatita, como substituto do enxerto ósseo em artrodeses do carpo em dois gatos.

\footnotetext{
${ }^{1}$ Pós-graduando em Cirurgia Veterinária, Faculdade de Ciências Agrárias e Veterinárias, Universidade Estadual Paulista (UNESP) Jaboticabal. ${ }^{2}$ Professor Assistente, Doutor, FCAV, UNESP Jaboticabal. Via de Acesso Prof. Paulo Donato Castellane Km 5, 14884-900, Jaboticabal, São Paulo, Brasil. E-mail: Padilha@fcav.unesp.br, Tel 5516 32092626, Fax: 551632024275. Autor para correspondência.

${ }^{3}$ Professor Doutor Assistente da Universidade Federal do Rio Grande do Sul.
} 


\section{RELATO DOS CASOS}

Caso 1: gato da raça Siamês, macho, não castrado, com 10 anos de idade, $5,0 \mathrm{~kg}$, apresentando afuncionalidade do membro torácico esquerdo, cujo tempo de ocorrência era desconhecido. Ao exame clínico, verificaram-se movimentos de hiperextensão, instabilidade radiocárpica, dor à palpação, abscesso próximo a região da articulação carpiana e ausência de alterações em nervos periféricos. Ao exame radiográfico, observou-se luxação radiocárpica, degeneração articular e osteoartrite.

Caso 2: gato sem raça definida, macho, não castrado, com oito meses de idade, $3,0 \mathrm{~kg}$, com o membro torácico direito afuncional, devido à união inadequada de fratura do rádio e ulna. Ao exame clínico, verificou-se contratura de tendões flexores na região da articulação radiocárpica, devido à aplicação da bandagem de Velpeau. Observou-se, ainda, rotação externa do olécrano, membro voltado para dentro e lesão cutânea na região articular. Não houve evidências de alterações em nervos periféricos.

As abordagens cirúrgicas das articulações foram realizadas conforme a descrição de PIERMATTEI (1993). Foi observado, no gato do caso 1, extensa fibrose, degeneração da cartilagem, não havendo sinais de infecção articular. As faces articulares, em ambos os casos, foram removidas com auxílio de curetas e freza elétrica a . Ato contínuo, adicionou-se a hidroxiapatita ${ }^{\mathrm{b}}$, em quantidade suficiente para preencher o defeito produzido pela curetagem.

As articulações foram anguladas em extensão, em ângulos de $175^{\circ}$ e $180^{\circ}$ graus, para os casos 1 e 2, respectivamente. A estabilização óssea, para ambos os casos, foi realizada mediante o emprego de fixador esquelético externo do tipo IIb, adaptado de PIERMATTEI \& FLO (1997), aplicando-se pino de rosca central de $2,5 \mathrm{~mm}$ no rádio proximal e pinos de $S c h a n z^{c}$ de 2,0mm, sendo um distal ao rádio e dois nos metatarsianos. Para o caso 2, foram utilizados três pinos de Schanz ${ }^{\mathrm{c}}$ nos metacarpianos, conectados por polimetilmetacrilato. No pós-cirúrgico, empregaramse bandagens acolchoadas para proteção do membro e do fixador e foi administrado cetoprofeno ${ }^{\mathrm{d}}(1,0 \mathrm{mg} /$ $\mathrm{kg}$ a cada 24 horas), pela via subcutânea, durante três dias; morfina ${ }^{\mathrm{e}}(0,1 \mathrm{mg} / \mathrm{kg})$, pela via intramuscular, durante três dias e ceptiofur ${ }^{\mathrm{f}}(2.2 \mathrm{mg} / \mathrm{kg}$, a cada 24 horas), pela via subcutânea, durante 10 dias consecutivos.

\section{RESULTADOS}

Durante o período de acompanhamento dos animais, não foram observadas formações de fístulas ou exsudato purulento. No pós-operatório imediato, notou-se edema intenso na extremidade do membro e em torno dos pinos, com decréscimo nos três dias subseqüentes. Compressas quentes e frias foram administradas como terapia coadjuvante. Já os fixadores esqueléticos externos foram bem tolerados pelos animais havendo apenas, permanecido ligeira rotação no membro no gato do caso 2 .

Nas análises radiográficas do caso 1 , aos 30 dias, a articulação apresentava sinais de proliferação óssea, mas, a fusão da articulação, só foi observada aos 60 dias (Figura 1a e 1d). Relativamente ao fixador esquelético externo, foi observado afrouxamento do pino de rosca central aos 60 dias. No caso 2, aos 30 dias observou-se secreção serosa em torno dos pinos de fixação com movimentação dos mesmos. A análise radiográfica, neste período, constatou o afrouxamento de todos os pinos de fixação, com lesões de reabsorção óssea em torno dos mesmos e evidenciado a união óssea da articulação (Figura 1c e $1 b)$.

Os implantes metálicos foram removidos após a confirmação da união óssea aos 60 e 30 dias respectivamente, para os casos 1 e 2 . No caso 1 , aos 90 dias, foi observado melhora no apoio, ausência de dor e na avaliação radiográfica, visibilizou-se a incorporação do excedente da hidroxiapatita e o fechamento dos orifícios referentes aos pinos de fixação. No caso 2, a despeito da correção cirúrgica, observou-se a persistência da rotação inicial do cotovelo, tendo sido recomendado ao proprietário procedimento de osteotomia corretiva do rádio e ulna.

\section{DISCUSSÃO}

A impossibilidade de correção das alterações resultantes, do processo degenerativo no caso 1 e da deformidade causada pela má-união da fratura do rádio e ulna, além da contratura dos tendões, no caso 2, tornaram as artrodeses necessárias, haja vista, que tal procedimento, conforme descrito por PENWICK (1987), LESSER (1993), JOHNSON (1995) e MOAK et al. (2000), é recomendado para processos que cursam com dor crônica, instabilidade e perda funcional carpiana, podendo restaurar a funcionalidade do membro.

Atribui-se o afrouxamento nos pinos de fixação óssea a complicações de sobrecarga aplicada sobre os mesmos (LESSER, 1993; OLMSTEAD et 


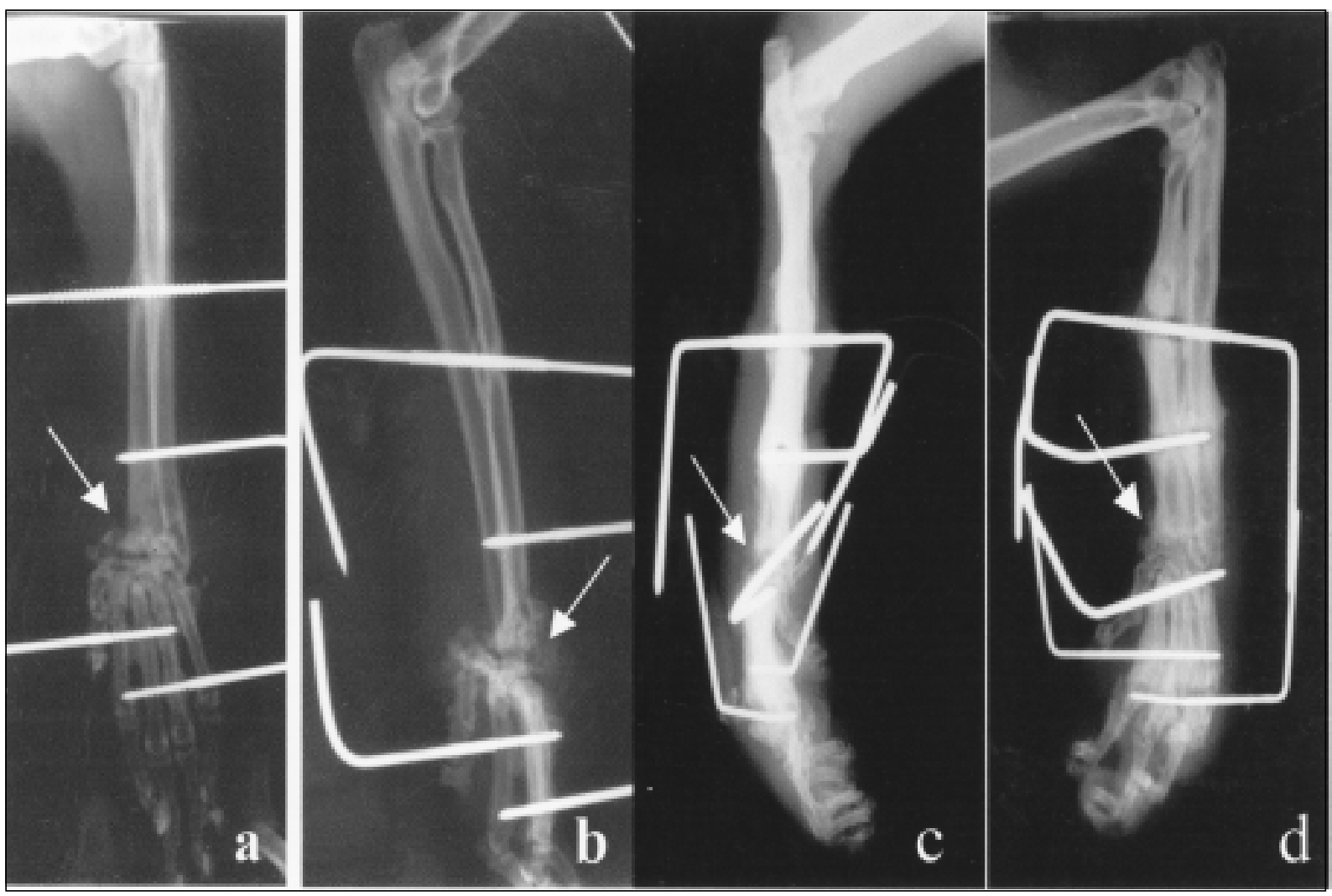

Figura 1 - Imagens radiográficas de artrodeses de carpo. Caso 1, após 60 dias do procedimento cirúrgico (a,b). Notar a disposição do pino de fixação óssea de rosca central aplicado no rádio, pinos de Schanz no rádio distal e nos metacarpianos. As setas indicam união óssea articular entre o osso rádio-cárpico e a face articular do rádio. Caso 2 aos 30 dias de pós-operatório (c, d). Notar fusão das superfícies articulares (setas) e lesões de reabsorção óssea em torno dos pinos de fixação.

al., 1995). LESSER (1993) salienta, ainda, que as estruturas mecânicas dos membros são arquitetadas para promover movimentação articular, desta forma, trabalham contra a imobilização no ponto de fixação.

A união óssea obtida aos 60 e 30 dias para os casos 1 e 2 , respectivamente, pode ser explicada pela diferença etária entre os animais, qualidade óssea, alimentação e atividade dos mesmos. No entanto, o tempo para a união óssea em artrodeses pode ser variável, pois, segundo JOHNSON (1995), pode ocorrer entre 4 e 8 semanas. Os tempos de união óssea obtidos nos dois casos foram semelhantes aos achados de JOHNSON \& BELENGER (1980) e GRUMADAS (1987), em artrodeses carpais experimentais em cães com utilização de enxerto ósseo. Tal fato pode ser atribuído a capacidade de osteotransdutividade da hidroxiapatita, que implicaria em biodegradação e substituição desta por novo osso de forma simultânea (CARRODEGUAS et al., 1999).

Embora sejam realizadas artrodeses em gatos na prática veterinária existem poucos relatos nesta espécie. MOAK et al. em 2000, ao relatar artrodeses de cotovelo em três gatos, utilizando enxerto ósseo autógeno verificaram, ainda, que os animais retornaram à normoatividade num período de 5 a 10 semanas não especificando o exato período de união óssea.

A hidroxiapatita foi preconizada para o preenchimento do espaço articular criado pela curetagem, tomando como prerrogativa a freqüente aplicação desta na medicina e odontologia e por esta ser um dos materiais mais biocompatíveis conhecidos (BLOKHUIS et al., 2000). Atribui-se esta característica à estrutura de cristal hexagonal e à similaridade com a fase mineral do tecido ósseo, além da porosidade da biocerâmica que influencia na sua osteocondutividade (BLOKHUIS et al., 2000), por servir de arcabouço para migração de vasos sangüíneos e deposição de novo osso. Fato este que também foi descrito por STEVESON (1993), PARKER (1995) e FOX (1983) relativamente aos enxertos ósseos. 
Apesar da extensa e freqüente aplicabilidade da hidroxiapatita na medicina humana e odontologia, na medicina veterinária a sua aplicação restringe-se, quase que exclusivamente, aos procedimentos experimentais, com a sua implantação em diversos sítios, mas, visando apenas à avaliação da sua biocompatibilidade. Esses estudos ressaltaram, também, resultados satisfatórios com relação à incorporação da hidroxiapatita, osteointegração e crescimento ósseo entre a cerâmica e o osso hospedeiro (DACULSI et al., 1989; FULLER et al., 1996; DELECRIN et al., 1997).

\section{CONCLUSÃO}

Tendo em vista os resultados obtidos e os dados da literatura sobre o comportamento biológico da hidroxiapatita, esta passa a apresentar-se como um substituto ósseo promissor na prática cirúrgica de pequenos animais.

\section{FONTES DE AQUISIÇÃO}

a Micro retifica elétrica Dremel ${ }^{\circledR}$

b Biosynth ${ }^{\circledR}$ (Biosynth LTDA, granulometria < 177 micra)

c. Pinos meios de rosca de perfil negativo. Biomecânica -SP

d Ketofen ${ }^{\circledR}$ Meryal

e Dimorf® Lab. Cristália

f Exenel® Shering Plung Veterinária

\section{AGRADECIMENTO}

A FAPESP processos no 00/07715-5 e 00/12655-1. Ao Prof. Dr. Júlio Carlos Canola pela execução dos exames radiográficos.

\section{REFERÊNCIAS BIBLIOGRÁFICAS}

BLOKHUIS, T. et al. Properties of calcium phosphate ceramics in relation to their in vivo behavior. J Trauma, v.48 n.1, p.179$86,2000$.

CARRODEGUAS, R.G., et al. Cimentos de alfa-fosfato tricálcico de fraguado doble. Revista CENIC - Ciências Químicas, v.30, n.3, p.153-158, 1999.

DACULSI, G. et al. Comparative study of bioactive calcium phosphate ceramics after implantation in spongy bone in dogs. Histologic, ultrastructural and electron probe microanalysis. Rev Chir Orthop, v.75, n2, p.65-71, 1989.

DELECRIN, J. et al. Influrence of local enviroment on incorporation of ceramic for lumbar fusion. Compairson of laminar and intertransverse sites in a canine model. Spine, v.22, n.15, p.1683-1689, 1997.
FOX, S.M. Cancellous bone grafting in the dog: overview. J Am Animal Hosp Assoc, v.20, p.840-848, 1983.

FULLER, D.A.; STEVENSON, S.; EMETY, S.E. The effects of internal fixation on calcium carbonate. Ceramic anterior spinal fusion in dogs. Spine, v.21, n.18, p.2131-2136, 1996.

GRUMADAS, C.E.S. Pan-artrodese do carpo na correção de instabilidade articular provocada experimentalmente por neurectomia do radial em caninos. 1987. $77 \mathrm{f}$. Dissertação (Mestrado em Medicina Veterinária) - Curso de Pós-graduação em Medicina Veterinária, Universidade Federal de Santa Maria.

JOHNSON, K.A. Arthrodesis. In: OLMSTEAD, M.L. Small animal orthopedics. 3.ed. Philadelphia : Mosby, 1995. p.503529.

JOHNSON, K.A; BELENGER, C.R. The effects of autologous bone grafting on bone healing after carpal arthrodesis in the dog. Vet Rec, v.107, n.9, p.126-132, 1980.

LEGEROS, R.Z. Calcium phosphate in oral biology and medicine. In: MYERS, H.M. Monographs in oral science. Basel : Karger, 1991. p.210.

LESSER, A.S. Arthrodesis. In: SLATTER, D. Textbook of small animal surgery. 2.ed. Philadelphia : Saunders, 1993. V.2, p.1888-1900.

MOAK, P.C, et al. Arthodesis of the elbow in three cats. Vet Comp Orthop Traumatol, v.13, n.3, p.149-153, 2000.

OLMSTAED, M.L.et al. Principles of fracture repair. In: OLMSTEAD, M.L. Small animal orthopedics. 3.ed. Philadelphia : Mosby, 1995. p.111-160.

PARKER, R.B. Injertos ósseos en cirurgía de pequeños animales. Waltham Focus, v.5, n.2, p.90-99, 1995.

PENWICK, R.C. Arthrodesis. Vet Clinics of North Am: Small Animal Pract, v.17, n.4, p.811-819, 1987.

PIERMATTEI, D.L. Approach to distal radious and carpus through a dorsal incision. In: An atlas of surgical approaches to the bones and joints of the dog and cat. 3.ed. Philadelphia: Saunders, 1993. p.204-205

PIERMATEI, D.L.; FLO, G. L. Arthrology. In: Handbolk of small animal orthopedics and fracture repair. 3.ed. Philadelphia : Saunders, 1997. p.170-200.

STEVESON, S. Bone grafting. In: SLATTER, D. Textbook of small animal surgery. 2.ed. Philadelphia : Saunders, 1993. V.2, p.1694-1703

TOOMBS, J.P. Transarticular application of external skeletal fixation. Vet Clinics of North Am: Small Animal Pract, v.22, n.1, p.181-194, 1987.

TURNER, T.M.; LIPOWITZ, A .J. Artrodese. In: BOJRAB J.M. Técnicas atuais em cirurgia de pequenos animais. 3.ed. São Paulo : Roca, 1996. p.775-793. 